AperTO - Archivio Istituzionale Open Access dell'Università di Torino

Shape controlled gold nanostructures on de-alloyed nanoporous gold with excellent SERS performance

This is a pre print version of the following article:

Original Citation:

Availability:

This version is available http://hdl.handle.net/2318/1691350

since 2019-03-13T16:30:35Z

Published version:

DOI:10.1016/j.cplett.2018.08.044

Terms of use:

Open Access

Anyone can freely access the full text of works made available as "Open Access". Works made available under a Creative Commons license can be used according to the terms and conditions of said license. Use of all other works requires consent of the right holder (author or publisher) if not exempted from copyright protection by the applicable law. 


\title{
Shape controlled gold nanostructures on de-alloyed nanoporous gold with excellent SERS performance
}

\author{
Yanpeng Xue ${ }^{1}$, Federico Scaglione ${ }^{1}$, Federica Celegato ${ }^{2}$, Pierre Denis ${ }^{3}$, Hans-Jörg Fecht ${ }^{3}$, \\ Paola Rizzi ${ }^{1}$, Livio Battezzati ${ }^{1}$ \\ ${ }^{1}$ Dipartimento di Chimica e Centro Interdipartimentale NIS (Nanostructured Surfaces and \\ Interfaces), Università di Torino, Via Pietro Giuria 7, 10125 Torino, Italy \\ ${ }^{2}$ Istituto Nazionale di Ricerca Metrologica (INRIM), Str. delle Cacce 91, 10135 Torino, Italy \\ ${ }^{3}$ Institute of Micro and Nanomaterials, University of Ulm, Albert-Einstein-Allee 47, \\ 89081 Ulm, Germany
}

*Corresponding Author:yxue@unito.it

\begin{abstract}
:
In this study, nanoporous gold (NPG) has been prepared by chemical de-alloying the $\mathrm{Au}$ based metallic glass precursor, $\mathrm{Au}_{20} \mathrm{Cu}_{48} \mathrm{Ag}_{7} \mathrm{Pd}_{5} \mathrm{Si}_{20}$ (at.\%), which was rapidly solidified from the melt in the form of ribbons. On the nanoporous gold, shape controlled gold nanostructures were electrodeposited by adjusting the applied potentials and deposition times. Using Rhodamine $6 \mathrm{G}(\mathrm{R} 6 \mathrm{G})$ as probe molecule, the surface enhanced Raman scattering (SERS) activities with different Au nanostructures on nanoporous gold were compared. The electrodeposited Au dendritic nanostructures on NPG after 3000s exhibited strongest SERS effect and high reproducibility. Using this best SERS active substrate for melamine sensing, the detection limit of $10^{-7} \mathrm{M}$ was achieved.
\end{abstract}

Key words: Nanoporous gold; Chemical de-alloying; Au electrodeposition; Raman spectroscopy; Melamine sensing.

\section{Introduction}

Since its discovery, Surface enhanced Raman scattering (SERS) has developed into one of the most versatile tools for providing high molecular specificity and sensitivity in identifying trace level of molecules in biological and chemical systems ${ }^{[1,2]}$. Single molecule detection can be achieved by using plasmonic metallic nanostructures to amplify the Raman scattering of targeted molecules ${ }^{[3,4]}$. It has been reported that the SERS enhancement is due to a chemical effect contributing for two-three orders of magnitude ${ }^{[5]}$, and a dramatic electromagnetic effect arising from the resonant excitation of localized surface plasmons on the metallic surface ${ }^{[6]}$. The local 
electromagnetic field is enhanced strongly around sharp edges or tips ${ }^{[7]}$, interparticle gaps ${ }^{[3,8]}$ and nanopores ${ }^{[9]}$, typically referred to as "hot spots". To date, extensive investigations have demonstrated that nanoporous metals with three dimensional (3D) bicontinuous structure prepared by de-alloying can be used as substrate for practical SERS applications ${ }^{[7,10]}$ because of possessing many active sites to increase the excitations of localized surface plasmons. Melamine has been illegally added to milk products to produce high protein content reading which cannot be distinguished from other proteins by the Kjeldahl or Dumas test ${ }^{[11]}$. Compared with conventional methods, SERS based detection techniques using molecularly imprinted polymers and hollow $\mathrm{Ag}-\mathrm{Au}$ alloys nanocubes as substrates have been developed for the sensitive trace analysis of melamine in food ${ }^{[12,13]}$. Recently, we reported that nanoporous gold was fabricated from a new $\mathrm{Au}_{20} \mathrm{Cu}_{48} \mathrm{Ag}_{7} \mathrm{Pd}_{5} \mathrm{Si}_{20}$ metallic glass precursor by chemical de-alloying and achieved a detection limit of $10^{-6} \mathrm{M}$ for melamine sensing ${ }^{[14]}$. However SERS mapping on the nanoporous gold indicates that the distribution of hot spots is not homogeneous.

Electrochemical deposition is a suitable method to prepare metal nanostructures with desired size and morphology on conductive substrates due to its simplicity, high controllability and scalability ${ }^{[15]}$. It is well known that the applied potential can determine the reduction rate of metal precursor, which influences the growth rate of metal nanostructures ${ }^{[16]}$. And the presence of precursor concentration gradient layer near the electrode surface can significantly influence the growth orientation of metal nanostructures. Many works have been done to prepare metal nanostructures for SERS-active substrates by electrochemical deposition, such as silver nanosheets ${ }^{[17]}$, triangular $\mathrm{Pd}$ rod nanostructures ${ }^{[18]}, \mathrm{Au}$ nanoparticles ${ }^{[19]}$ and thin films ${ }^{[20]}$. On nanoporous gold, Yang et al. prepared silver nanoparticles by electroless deposition and observed $10^{4}$ times SERS improvement compared to the as-prepared NPG films, which is attributed to the abundant Raman-active nanogaps constructed adjacent nanoparticles and also by the NPG ligaments and adhered nanoparticles ${ }^{[21]}$. Fu et al. fabricated $\mathrm{Au} / \mathrm{SnO} / \mathrm{Ag}$ heterogeneous films by the same technique to achieve ultralow detection limits for both the resonant rhodamine $6 \mathrm{G}\left(10^{-15} \mathrm{M}\right)$ and the non-resonant and low cross-section cysteine $\left(10^{-10} \mathrm{M}\right)^{[22]}$. Zhang et al. achieved sharp gold nano-cones on nanoporous gold de-alloyed form the $\mathrm{Au}_{35} \mathrm{Ag}_{65}($ at.\%) alloy, which demonstrates high SERS enhancement with a detection limit of $10^{-12} \mathrm{M}$ for Rhodamine $6 \mathrm{G}$ as probe molecule ${ }^{[7]}$.

In the present study, we first time report three dimensional $\mathrm{Au}$ dendritic nanostructures on nanoporous gold, which are facilely fabricated by electrochemical deposition, as uniform SERS active substrate for melamine sensing. First nanoporous gold was prepared by chemically de-alloying from Au based metallic glass precursor in the form of ribbon. On the nanoporous gold surface, the shape controlled gold nanostructures were electrodeposited by adjusting the applied potentials and deposition times. Using Rhodamine 6G (R6G) as probe molecule, the SERS activities with different $\mathrm{Au}$ nanostructures on nanoporous gold were compared. Finally, sensing of 
melamine in aqueous solution is successfully attempted using the best SERS active substrate.

\section{Experimental:}

$\mathrm{A} \mathrm{Au}_{20} \mathrm{Cu}_{48} \mathrm{Ag}_{7} \mathrm{Pd}_{5} \mathrm{Si}_{20}$ (at. \%) master alloy was prepared by arc melting the pure elements (Au: 99.99\%, Ag, Cu, Pd: 99.99\%, Si: 99.9995\%) in Ti-gettered Argon atmosphere. The ingot was rapidly solidified onto a rotating copper wheel at a linear speed of $25 \mathrm{~m} / \mathrm{s}$ by the melt-spinning process. The thickness of the resulting ribbon is $20 \mu \mathrm{m}$ and the width is $2 \mathrm{~mm}$.

Nanoporous gold(NPG) was prepared by chemical de-alloying of the as-spun ribbons in $10 \mathrm{M} \mathrm{HNO}_{3}$ and $0.5 \mathrm{M} \mathrm{HF}$ solution at $70^{\circ} \mathrm{C}$ for 4 hours. To achieve $\mathrm{Au}$ electrodeposition, a standard three electrode configuration was used: the de-alloyed NPG sample as working electrode, a $\mathrm{Ag} / \mathrm{AgCl}$ electrode as reference and a platinum sheet as counter electrode respectively. Au electrodeposition was conducted in the aqueous solution of $1 \mathrm{mM} \mathrm{HAuCl}_{4}$ and $0.5 \mathrm{M} \mathrm{H}_{2} \mathrm{SO}_{4}$ as the supporting electrolyte ${ }^{[23]}$. All chemicals used were analytical grade and used without further purification.

The surface morphology of de-alloyed samples was observed by scanning electron microscopy (SEM) and their compositions were checked by energy dispersive X-ray spectroscopy (EDS) after Co calibration. The structure of the materials was studied by X-ray diffraction (XRD) using $\mathrm{Cu} \mathrm{K}_{\alpha}$ wavelength in Bragg-Brentano mode. The structure of the electrodeposited $\mathrm{Au}$ thin film was characterized by glancing angle X-ray diffraction (GAXRD, Philips PW3830), measured with a glancing angle of 0.8 under $\mathrm{Cu} \mathrm{K} \alpha$ radiation at $40 \mathrm{kV}$.

Micro-Raman measurements were performed with a Renishaw inVia Raman Microscope using $785 \mathrm{~nm}$ laser line with an acquisition time of $20 \mathrm{~s}, 0.4 \mathrm{~mW}$ power at the sample and a $50 \times$ ULWD objective; Rhodamine $6 \mathrm{G}(\mathrm{R} 6 \mathrm{G})$ was chosen as SERS probe molecule. Prior to SERS experiments, the samples were cleaned in concentrated nitric acid for 5 minutes and rinsed several times in de-ionized water. Then, the samples were immersed in aqueous solution of Rhodamine $6 \mathrm{G}$ with concentration of $10^{-6} \mathrm{M}$ for one night, enabling the probe molecule to be adsorbed on the surface. For melamine sensing, the de-alloyed samples were immersed in the aqueous solution of melamine with concentrations from $10^{-7} \mathrm{M}$ to $10^{-3} \mathrm{M}$ for 1 night. All solutions were prepared from chemical grade reagents and de-ionized water. The Raman band of silicon wafer at $520 \mathrm{~cm}^{-1}$ was used to calibrate the spectrometer.

\section{Results and discussion}

Nanoporous gold (NPG) was prepared by chemical de-alloying in a mixture of 10 $\mathrm{M} \mathrm{HNO}_{3}$ plus $0.5 \mathrm{M} \mathrm{HF}$ at $70^{\circ} \mathrm{C}$ for 4 hours from the $\mathrm{Au}_{20} \mathrm{Cu}_{48} \mathrm{Ag}_{7} \mathrm{Pd}_{5} \mathrm{Si}_{20}$ metallic glass precursor. During the de-alloying process, accompanying with the dissolution of the less noble elements, $\mathrm{Au}$ adatoms reorganize into interconnected ligaments separated by open pores by surface diffusion ${ }^{[24]}$. After chemical de-alloying, the SEM image of NPG reveals a uniform bicontinuous nanoporous structure which is 
composed of nanopores and ligaments with average size of around $60 \mathrm{~nm}$ (Fig 1a). And the XRD pattern demonstrates the formation of face-centered cubic (fcc) Au from the amorphous state(Fig S1).

\subsection{Au electrodeposition under different potentials}

The Au nanostructures on de-alloyed NPG were achieved by electrochemical deposition from a solution of $1 \mathrm{mM} \mathrm{HAuCl}_{4}$ and $0.5 \mathrm{M} \mathrm{H}_{2} \mathrm{SO}_{4}$ as the supporting electrolyte $^{[23]}$. During the electrodeposition process, the applied potentials can influence the reduction rate of metal ions and the thickness of the precursor concentration gradient layer, thus determining the morphologies of final products. Therefore the effect of deposition potentials on the morphology of Au nanostructures was examined first. Fig.1 shows the SEM images of the deposited gold nanostructures on NPG at room temperature for 3000 s under the applied potential of $0.24 \mathrm{~V},-0.04 \mathrm{~V}$ and $-0.34 \mathrm{~V}$ versus $\mathrm{Ag} / \mathrm{AgCl}$ reference electrode, respectively. Under the applied potential of $0.24 \mathrm{~V}$, hierarchical waxberry like nanostructures with uniform diameter of around $180 \mathrm{~nm}$ were obtained(Fig 1b). Many tiny Au nanosheets cover the top of waxberry like nanostructures. The Au ligaments underneath can still be observed through the voids among the waxberry like structures. Under the applied potential of $-0.04 \mathrm{~V}$, Au three dimensional dendritic nanostructures with preferred orientation were formed. The formation of Au nanodendrites were attributed to the diffusion limitation of $\mathrm{Au}$ ions on the growing nanostructures under this applied potential. From the high magnification of Au nanodendrites image (Fig 1e), most of the dendrites are standing vertically to the de-alloyed NPG with height of around 70nm and a sharp tip apex of around $15 \mathrm{~nm}$. The height of nanodendrites is different probably due to the diffusion fronts of Au ions to the growing nanostructures overlap, which results in different growth rates. The Glancing angle XRD pattern of nanodendrites contains only reflections associated to face-centered cubic Au (Fig. S1c), which confirms the pure Au formation after the electrodeposition process. By shifting negatively the applied potential to $-0.34 \mathrm{~V}$, irregular polyhedral Au nanoparticles with a mean size of $30 \mathrm{~nm}$ were obtained. The high overpotential reduces the influence of the diffusion limited aggregation process on

different crystallographic facets, leading to the isotropic growth of different crystallographic facets. 

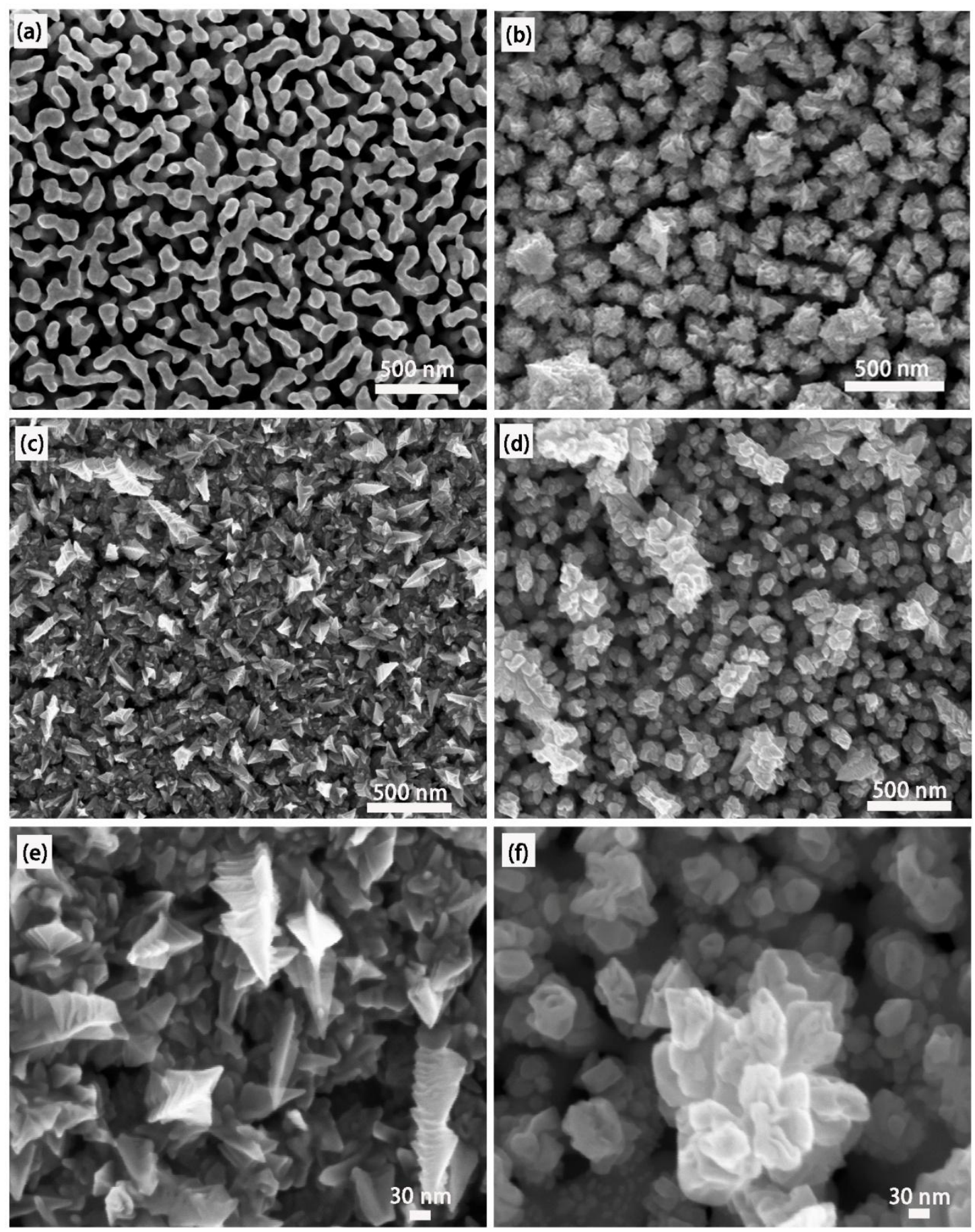

Figure 1. SEM images of (a) sample after chemically de-alloyed in $10 \mathrm{M} \mathrm{HNO}_{3}+0.5 \mathrm{M}$ $\mathrm{HF}$ at $70^{\circ} \mathrm{C}$ for 4 hours and samples Au deposited on nanoporous gold in $1 \mathrm{mM} \mathrm{HAuCl}_{4}$ $+0.5 \mathrm{M} \mathrm{H}_{2} \mathrm{SO}_{4}$ at room temperature for $3000 \mathrm{~s}$ under different potentials (b) $0.24 \mathrm{~V}$, (c) $-0.04 \mathrm{~V}$ and $(\mathrm{d})-0.34 \mathrm{~V}$. (e) and (f) are the enlargements of (c) and (d) respectively.

\subsection{Au electrodeposition under different times}

To further understand the growth mechanism of $\mathrm{Au}$ dendritic nanostructures on nanoporous gold, we performed chronoamperometry experiments at the applied potential of $-0.04 \mathrm{~V}$ for electrodeposition time of $100,300,1000$ and 2000s. The 
obtained surface morphologies of Au deposits are shown in Fig. 2. For short electrodeposition time corresponding to 100s, tiny Au nanoparticles are formed on the top surface of Au ligaments(Fig. 2a). And there are no tiny Au nanoparticles formed on the ligaments underneath the top surface, which can be due to the mass transport limitations under this deposition conditions. Usually at the initial electrodeposition process, the gold deposits will nucleate at the relative active sites on the ligaments such as steps and grain boundaries which were formed during the de-alloying process. With the increase of deposition time to 300s, Au nanoparticles connected with each other, coalesce and cover the top Au ligaments surface(Fig. 2b). And small Au nanoplates with random orientation grow on the top of connected Au nanoparticles. Increasing the electrodeposition time to 1000s, the Au nanoplates cover completely the top surface of $\mathrm{Au}$ ligaments and we can still observe the Au ligaments underneath (Fig. 2c). When the electrodeposition time increases to 2000s, three dimensional Au nanodendrites grow on the top of connected nanoplates (Fig.2d). From the cross section of sample, the $\mathrm{Au}$ nanodendrites only formed on the top of Au ligaments. And the nanoporous gold underneath remains a network of ligament connected into a 3D nanoporous structure. At the initial stage of electrodeposition process, there is no significant concentration gradient of $\mathrm{Au}$ precursor at the electrode/electrolyte interface. Therefore $\mathrm{Au}$ nanoparticles are formed on the ligaments randomly, especially at the relative active sites on the ligaments such as steps and grain boundaries. As the electrodeposition process proceeds, the $\mathrm{Au}^{3+}$ concentration gradient layer starts to form. At this time, $\mathrm{Au}$ nanoplates with random orientation are obtained on the top surface of Au ligaments. Increasing the deposition time, the concentration gradient layer becomes thicker, the $\mathrm{Au}$ dendritic nanostructures are formed which protrude into the bulk solution to access more $\mathrm{Au}^{3+}$ ions. The Au nanoparticles on the ligaments surface will grow slower and slower until depletion of metal precursor near the ligaments. Finally, three dimensional Au dendritic nanostructures are formed vertically on the Au ligaments surface. 

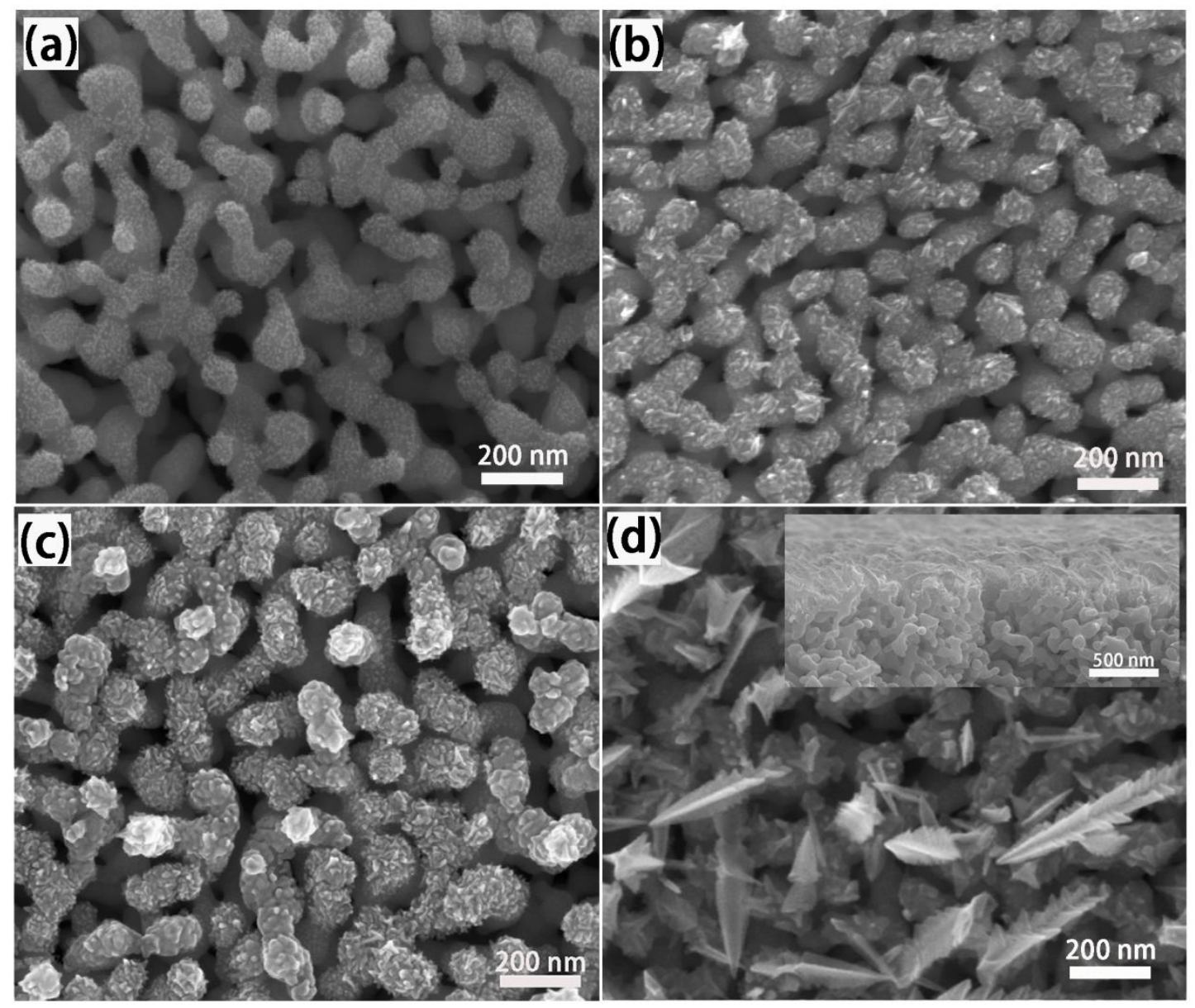

Figure 2. SEM images of samples Au deposited in $1 \mathrm{mM} \mathrm{HAuCl} 4+0.5 \mathrm{M} \mathrm{H}_{2} \mathrm{SO}_{4}$ at room temperature under applied potential of $-0.04 \mathrm{~V}$ for different time (a) $100 \mathrm{~s}$, (b) $300 \mathrm{~s}$, (c) 1000s and (d) 2000s. Inset shows the cross section of sample.

\subsection{SERS effect of nanoporous gold + electrochemical deposition}

In order to check the SERS capability of $\mathrm{Au}$ nanostructures with different morphologies, samples electrodeposited under different applied potentials and deposition times were tested. Figure 3a shows the SERS spectra of deposited samples under different applied potentials after immersing them for one night in aqueous solution of Rhodamine $6 \mathrm{G}$ with concentrations of $10^{-6} \mathrm{M}$. All SERS spectra display the characteristic peaks of Rhodamine $6 \mathrm{G}$ in good agreement with the literatures ${ }^{[7,25]}$. The characteristic peaks of R6G at Raman shift of 1652, 1511 and $1364 \mathrm{~cm}^{-1}$, which are attributed to the symmetric modes of the in-plane C-C stretching vibrations. The Raman band at 1312 and $1186 \mathrm{~cm}^{-1}$ are associated with the $\mathrm{N}-\mathrm{H}$ and $\mathrm{C}-\mathrm{H}$ in-plane bend vibrations respectively. And the Raman band at 774 and $612 \mathrm{~cm}^{-1}$ are assigned to the $\mathrm{C}-\mathrm{H}$ out-of-plane and C-C-C ring in-plane bending vibrations. The SERS activities changes with different $\mathrm{Au}$ nanostructures deposited on the top surface of nanoporous Au ligaments by varying the applied potentials. Compared with the SERS intensity of nanoporous gold, the waxberry-like $\mathrm{Au}$ nanostructure prepared under the applied potential of $0.24 \mathrm{~V}$ exhibits the same order of magnitude. The irregular polyhedral $\mathrm{Au}$ 
nanoparticles prepared under the applied potential of $-0.34 \mathrm{~V}$ shows 1.8 times higher comparing with that of nanoporous gold while the three dimensional Au dendritic nanostructures obtained under applied potential of $-0.04 \mathrm{~V}$ shows 6 times higher than that of nanoporous gold. It is well know that the sharp tips can act as 'hot spots', where the local electromagnetic fields are dramatically amplified due to the resonant excitation of localized surface plasmons.

Under the applied potential of $-0.04 \mathrm{~V}$, we further investigated the influence of electrodeposition times on the SERS activity using R6G as the probe molecule. Figure $3 b$ shows the SERS spectra of samples deposited for different times under the same Raman measurements. The SERS intensity increases with electrodeposition times. At the initial electrodeposition process, tiny nanoparticles or small nanoplates deposited on the ligaments of top surface and the improvement of SERS intensity is not obvious. After the formation of $\mathrm{Au}$ dendritic nanostructures electrodeposited for 2000s, the SERS intensity doubled compared with that of nanoporous gold. By increasing the deposition time to 3000s, the SERS activity of Au dendritic nanostructures exhibits 6 times stronger than that of nanoporous gold. In order to test the reproducibility of the prepared Au dendritic nanostructure, SERS spectra were collected at different locations on one substrate and between different substrates (Fig. S2) resulting in a very similar peak position and intensity of the SERS spectra.

The enhancement of SERS effect on nanoporous gold mainly arises from local electromagnetic field enhancement caused by the resonant excitation of localized surface plasmons around nanosized ligaments. Also, the electromagnetic coupling effect between adjacent gold ligaments may further enhance the localized field intensity, leading to improvement of the SERS enhancement ${ }^{[26]}$. However, the distribution of hot spots on nanoporous gold is not homogeneous. In order to achieve the homogeneous distribution of hot spots, different Au nanostructures were prepared by electrochemical deposition on the top surface of nanoporous gold. Around these nanostructures, the local electromagnetic field is enhanced strongly and the SERS intensity becomes stronger, especially for the three dimensional Au dendritic nanostructures with sharp tips. 

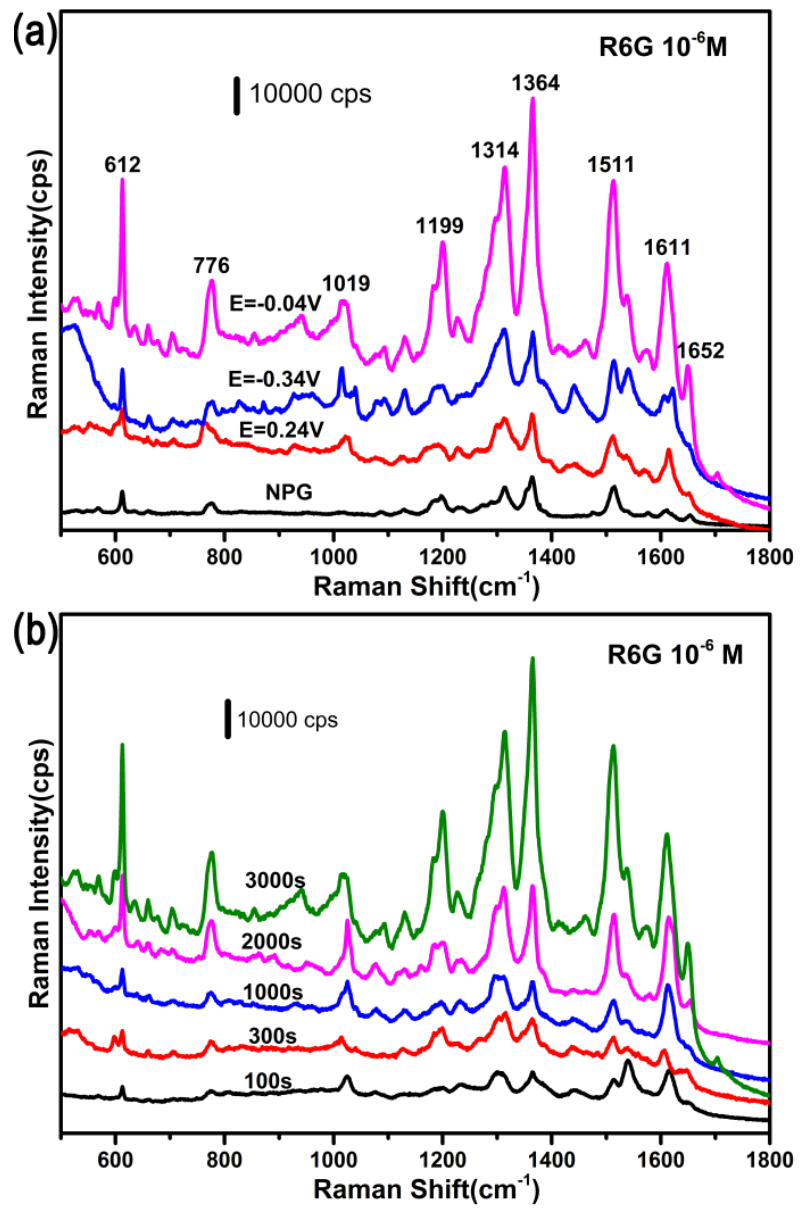

Figure 3. (a) SERS spectra of R6G with concentration of $10^{-6} \mathrm{M}$ on NPG and samples deposited under different applied potentials. (b) SERS spectra of R6G with concentration of $10^{-6} \mathrm{M}$ on samples deposited for different times.

\subsection{Detection of melamine using NPG+electrochemical deposition}

To illustrate the potential applications of the prepared Au dendritic nanostructure on nanoporous gold, the different concentrations of melamine ranging from $10^{-7}$ to $10^{-3} \mathrm{M}$ in aqueous solution were detected (Fig.4a). For melamine powder, the characteristic peak of melamine is around at $677 \mathrm{~cm}^{-1}$ which is caused by the in-plane deformation of the triazine ring with the vibration of the amino nitrogen atoms. In the previous work we showed that on nanoporous gold the characteristic peak of melamine in the aqueous solution at $710 \mathrm{~cm}^{-1}$ is red-shifted by $33 \mathrm{~cm}^{-1}$ compared to that of melamine powder, which is in agreement with the literatures on gold nanoparticles ${ }^{[27,28]}$ and gold nanofinger surfaces ${ }^{[29]}$. It is showed that the characteristic peak of melamine can still be clearly observed when the melamine concentration decreases to $10^{-7} \mathrm{M}$. The SERS intensity at $710 \mathrm{~cm}^{-1}$ versus melamine concentration is plotted in Fig. 4(b). The result shows that the SERS intensities of melamine decrease monotonously with the decrease of melamine concentration. To evaluate the homogeneity of the prepared Au dendritic nanostructure on nanoporous gold, Raman mapping of $48 \times 48 \mu \mathrm{m}^{2}$ by lateral step of 4 $\mu \mathrm{m}$ was collected. Figure 4(c) shows a SERS intensity mapping image with melamine 
concentration of $10^{-5} \mathrm{M}$, which clearly indicates the presence of "hot spots". In contrast with as-prepared nanoporous gold, the "hot spots" on the Au dendritic nanostructure are high density and distributed homogeneously. Recently Zhang et al. showed that the detection limits of melamine down to $10^{-7} \mathrm{M}$ were achieved on three-dimensional sunflower-like nanoarrays decorated with Ag nanoparticles ${ }^{[30]}$. Huang et al. pushed the detection limits of melamine down to $10^{-10} \mathrm{M}$ on silver nanoparticles decorated zinc oxide/silicon heterostructured nanomace arrays ${ }^{[31]}$. Compared with those substrates, the fabrication process of our substrate is simple, easy-to-control and high reproducibility.
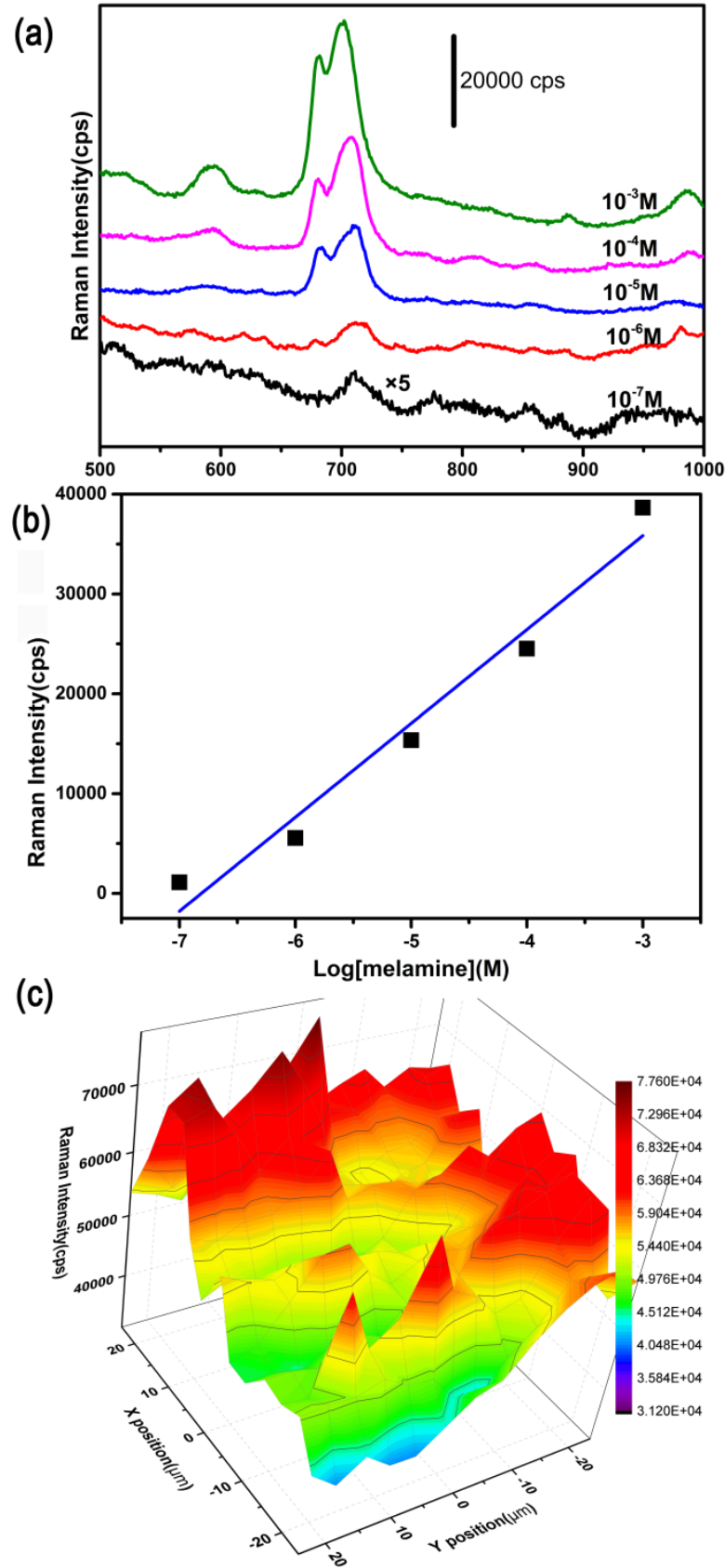

Figure 4. (a) SERS spectra of melamine at different concentrations on de-alloyed sample. (b) Raman intensity at $710 \mathrm{~cm}^{-1}$ versus melamine concentration (in logarithmic scale) ranging from $10^{-7}$ $\mathrm{M}$ to $10^{-3} \mathrm{M}$. (c) SERS intensity mapping image of $48 \times 48 \mu \mathrm{m}^{2}$ by lateral step of $4 \mu \mathrm{m}$ with melamine concentration of $10^{-5} \mathrm{M}$ based on characteristic peak at $710 \mathrm{~cm}^{-1}$. The laser wavelength was $785 \mathrm{~nm}$ 
with an acquisition time of $20 \mathrm{~s}$.

\section{Conclusion}

In this study, nanoporous gold has been prepared by chemical de-alloying of $\mathrm{Au}$ based metallic glass precursor, $\mathrm{Au}_{20} \mathrm{Cu}_{48} \mathrm{Ag}_{7} \mathrm{Pd}_{5} \mathrm{Si}_{20}$ (at.\%), which was rapidly solidified from the melt in the form of ribbons. The shape controlled gold nanostructures such as hierarchical waxberry like nanostructures, Au nanodendrites, polyhedral nanoparticles were electrodeposited under the applied potential of $0.24 \mathrm{~V},-0.04 \mathrm{~V}$ and $-0.34 \mathrm{~V}$, respectively. The formation of Au nanodendrites with specific orientation is attributed to the diffusion limited aggregation process by investigating the effect of electrodepsition times. By comparing the SERS activities with different $\mathrm{Au}$ nanostructures on nanoporous gold, we found that the electrodeposited Au dendritic nanostructures on NPG after 3000s exhibited strongest SERS effect. Using this best SERS active substrate for melamine sensing, the detection limit of $10^{-7} \mathrm{M}$ was achieved which shows potential application in food safety inspection and life science.

\section{Acknowledgements}

Dr Alessandro Damin worked in Centro Interdipartimentale NIS (Nanostructured Surfaces and Interfaces) from Università di Torino is kindly acknowledged for the SERS experiments. This work was supported by the funding scheme of the European Commission, Marie Curie Actions - Initial Training Networks (ITN) in the frame of the project VitriMetTech-Vitrified Metals Technologies and Applications in Devices and Chemistry, 607080 FP7-PEOPLE-2013-ITN and also by BINGO Project-Torino_call2014_L2_146 and Compagnia di San Paolo.

\section{References}

[1] S. Schlücker, Angew. Chemie - Int. Ed. 2014, 53, 4756.

[2] L. A. Lane, X. Qian, S. Nie, Chem. Rev. 2015, 115, 10489.

[3] S. Nie, S. R. Emory, Science (80-. ). 1997, 275, 1102.

[4] K. Kneipp, Y. Wang, H. Kneipp, L. T. Perelman, I. Itzkan, R. R. Dasari, M. S. Feld, Phys. Rev. Lett. 1997, 78, 1667.

[5] S. M. Morton, L. Jensen, J. Am. Chem. Soc. 2009, 131, 4090.

[6] N. P. W. Pieczonka, R. F. Aroca, Chem. Soc. Rev. 2008, 37, 946.

[7] L. Zhang, H. Liu, L. Chen, P. Guan, B. Chen, T. Fujita, Y. Yamaguchi, H. Iwasaki, Q.-K. Xue, M. Chen, RSC Adv. 2016, 6, 2882.

[8] D.-K. Lim, K.-S. Jeon, J.-H. Hwang, H. Kim, S. Kwon, Y. D. Suh, J.-M. Nam, Nat. Nanotechnol. 2011, 6, 452.

[9] X. Zhang, Y. Zheng, X. Liu, L. Wei, J. Dai, D. Y. Lei, D. R. MacFarlane, Adv. Mater. 2015, 27, 1090.

[10] H. Liu, L. Zhang, X. Lang, Y. Yamaguchi, H. Iwasaki, Y. Inouye, Q. Xue, M. Chen, Sci. Rep. 2011, 1, 1.

[11] M. Thompson, L. Owen, K. Wilkinson, R. Wood, A. Damant, Analyst 2002, 127, 1666.

[12] Y. Hu, S. Feng, F. Gao, E. C. Y. Li-chan, E. Grant, X. Lu, FOOD Chem. 2015, 176, 123.

[13] J. Li, Y. Yin, D. Qin, J. Mater. Chem. C 2014, 2, 9934.

[14] Y. Xue, F. Scaglione, P. Rizzi, L. Battezzati, Appl. Surf. Sci. 2017, 426, 1113.

[15] A. Taleb, X. Yanpeng, S. Munteanu, F. Kanoufi, P. Dubot, Electrochim. Acta 2013, 88, 621. 
[16] Q. Wu, P. Diao, J. Sun, T. Jin, D. Xu, M. Xiang, J. Phys. Chem. C 2015, 119, 20709.

[17] Y. Xia, Y. Wu, T. Hang, J. Chang, M. Li, Langmuir 2016, 32, 3385.

[18] S. Choi, H. Jeong, K. H. Choi, J. Y. Song, J. Kim, ACS Appl. Mater. Interfaces 2014, 6, 3002.

[19] J. Wang, X. Cao, L. Li, T. Li, R. Wang, J. Phys. Chem. C 2013, 117, 15817.

[20] J. Elias, M. Gizowska, P. Brodard, R. Widmer, Y. DeHazan, T. Graule, J. Michler, L. Philippe, Nanotechnology 2012, 23, 255705.

[21] M. Yang, L. Zhang, B. Chen, Z. Wang, C. Chen, Nanotechnology 2017, 28, 55301.

[22] H.-Y. Fu, X.-Y. Lang, C. Hou, Z. Wen, Y.-F. Zhu, M. Zhao, J.-C. Li, W.-T. Zheng, Y.-B. Liu, Q. Jiang, J. Mater. Chem. C 2014, 2, 7216.

[23] T.-H. Lin, C.-W. Lin, H.-H. Liu, J.-T. Sheu, W.-H. Hung, Chem. Commun. (Camb). 2011, 47, 2044.

[24] J. Erlebacher, M. J. Aziz, a Karma, N. Dimitrov, K. Sieradzki, Nature 2001, 410, 450.

[25] H.-C. Chen, T.-C. Hsu, Y.-C. Liu, K.-H. Yang, RSC Adv. 2014, 4, 10553.

[26] X. Lang, P. Guan, L. Zhang, T. Fujita, M. Chen, J. Phys. Chem. C 2009, 113, 10956.

[27] A. M. Giovannozzi, F. Rolle, M. Sega, M. C. Abete, D. Marchis, A. M. Rossi, Food Chem. 2014, $159,250$.

[28] Z. Guo, Z. Cheng, R. Li, L. Chen, H. Lv, B. Zhao, J. Choo, Talanta 2014, 122, 80.

[29] A. Kim, S. J. Barcelo, R. S. Williams, Z. Y. Li, Anal. Chem. 2012, 84, 9303.

[30] X. Zhang, X. Xiao, Z. Dai, W. Wu, X. Zhang, L. Fu, C. Jiang, Nanoscale 2017, 3114.

[31] J. Huang, F. Chen, Q. Zhang, Y. Zhan, D. Ma, K. Xu, Y. Zhao, ACS Appl. Mater. Interfaces 2015, 7, 5725 . 